\title{
Early discharging patients with chest pain using EDACS-ADP and COMPASS-MI risk predictors
}

\author{
Göksu Bozdereli Berikol ${ }^{1}$ [D $\cdot$ Hakan Aydın ${ }^{1} \cdot$ Halil Doğan $^{1}$
}

Received: 1 September 2021 / Accepted: 28 January 2022 / Published online: 8 February 2022

(c) Springer Japan KK, part of Springer Nature 2022

\begin{abstract}
Deciding on the early discharge of low-risk patients with chest pain is still controversial in emergency care. Beyond the validated tools for risk assessment, high sensitive troponin levels on admission, whether to take the next serial sampling or when to take are the main issues affecting the unnecessary follow-ups that lead to the emergency crowd. We aimed to investigate the prediction performance of emergency department assessment of chest pain score and accelerated diagnostic protocol (EDACS-ADP) and calculation of MI risk probabilities to manage patients with suspicion of myocardial infarction (COMPASS-MI). We conducted a prospective cross-sectional study that included patients with chest pain followed-up in the emergency department with a serial troponin sampling. We calculated the performance tests of the risk scores after recording the patients' risk factors, chest pain types, troponin levels as defined in the risk assessment tools. Nine hundred eleven patients were included in the study. Thirty-eight patients had significant adverse cardiovascular events (MACE) within 30 days. Patients with a not-low-risk score at EDACS-ADP had a 3.975 (95\% CI 2.136-7.396) fold higher risk of MACE than the patients with low-risk EDACS-ADP, and the absolute risk increase was 7.3\%. Patients with high-risk late-stage risk in COMPASS-MI had a 3.581 (95\% CI 1.660-7.726) fold higher risk of MACE than those with low-risk late-stage risk in COMPASS-MI, and absolute risk increase was $4.6 \%$. We found EDACS-ADP and COMPASS-MI at a late time point $(2 \mathrm{~h}$ hsTnI) with a high negative predictive value as a risk assessment tool for discharging chest pain patients.
\end{abstract}

Keywords Acute coronary syndrome $\cdot$ Chest pain $\cdot$ Patient discharge $\cdot$ Troponin I

\section{Introduction:}

There are 7-8 million chest pain visits per year in the United States, approximately $10-15 \%$ of the acute coronary syndrome (ACS), of which $1-2 \%$ of them are misdiagnosed and discharged [1,2]. Validated risk scores are occasionally used to avoid misdiagnosis and to predict the cardiac mortality of patients presented to the emergency department with chest pain [2-6]. Besides their superiorities, all these scores help emergency physicians decide whether these patients could discharged from the emergency department safely or would have major advanced cardiac events (MACE) in the future. In the calculation of these scores, the patient's

Göksu Bozdereli Berikol gokxsu@hotmail.com

1 Department of Emergency Medicine, İstanbul Bakırköy Dr Sadi Konuk Training and Research Hospital, Zuhuratbaba Mah, Dr. Tevfik Sağlam Cd No:11, 34147 Bakırköy/Istanbul, Turkey age, risk factors for atherosclerosis and coronary artery disease (family history, gender, smoking, hypertension, hypercholesterolemia, diabetes, obesity), known coronary artery stenosis, aspirin use, the severity of angina, changes in electrocardiography (ECG) and changes in biomarkers as high sensitive cardiac troponin level are majorly used [4-6]. Especially abnormal findings in ECG and cardiac biomarkers or their changes in the ED help emergency physicians' decision-making in ED on discharging or further follow-up.

One of the significant sensitive biomarkers for acute myocardial infarction (AMI) is high-sensitivity cardiac Troponin I (hsTnI), frequently used in the routine of chest pain in the emergency department, is used alone or in conjunction with scoring systems, especially in deciding the discharge of patients with low and medium risk chest pain. High precision and sensitivity of the biomarker allow physicians to rule-out AMI and serial troponin measurements to ensure discharge of the patients with low risk [7]. Very low first troponin levels are predictors of very low 30-day MACE and death [7-9]. There is a need for serial troponin testing 
for patients with chest pain less than $2 \mathrm{~h}$ due to the low NPV values of $\mathrm{hsTnI}<5 \mathrm{ng} / \mathrm{L}[7,8]$. These patients are still in debate for discharging from emergency departments.

Besides the studies carried out to decide on the early discharge of patients with chest pain via $0-h, 1-h$, and 2-h high-sensitive Troponin (hsTn) [7], Emergency Department Assessment of Chest Pain and Accelerated Diagnostic Protocol (EDACS-ADP) have been put forward to determine the follow-up needs of patients with low-risk chest pain both with the aid of risk factors and biomarkers [10].

The Calculation Of MI risk probabilities to Manage Patients with SuSpicion of Myocardial Infarction (COMPASS-MI); a new method to measure the change in troponin value the patient's mortality within 30 days in patients with low-risk chest pain, was created [6].

Studies have been trying to find the best combinations of these variables to minimize the duration of ED stay and bed use without missing the actual AMI patients.

This study aimed to investigate the performances of ADP and COMPASS-MI risk predictors regarding the 30-dayMACE of patients with low-risk chest pain in the emergency department.

\section{Methods}

\section{Study design}

Our study is a single-center prospective cross-sectional study and was conducted after the local ethical approval of the institution following the Helsinki statement. The study period was between December 1, 2019, and July 1, 2020, in a third-level training and research hospital. The patients who gave consent to participate in the study and met the inclusion criteria were included (Fig. 1).

\section{Study settings and population}

The study was conducted in a tertiary hospital (training and research hospital), which has 350,000 patient ED visits per annum. The emergency department was using a three-level triage algorithm defined by the Ministry of Health. The patients were first evaluated in the triage, with vital signs, ECG, and a short history of the chief complaints.

\section{Study protocol}

\section{Patient selection}

After the ethical approval, all the patients admitted to our emergency department with chest pain are included in the study consecutively. The patients with symptoms suggestive of acute coronary syndrome without chest pain (shortness of breath, syncope, epigastric pain) not had not been included in the study. All the patients who were investigated for the suspected acute coronary syndrome and had the other chest pain diagnosis discarded (as pneumothorax, pulmonary embolism, pneumonia) are included in the analysis. Figure 1 shows the selection of the patients.

Inclusion criteria in the study:

- Patients $\geq 18$ years with chest pain and investigated for AMI.

- Patients with normal vital signs.

- Patients who gave informed consent to participate in the study.

Exclusion criteria from the study:

- Patients whose chest pain continues or increases during follow-up in the ED.

- Patients with traumatic chest pain.

- Patients with suspected or detected COVID-19 in the study period.

- Patients requiring hospitalization for non-cardiac, medi$\mathrm{cal} /$ surgical/psychiatric reasons.

- Patients with other diagnoses as ischemic/hemorrhagic cerebrovascular event, acute renal failure, requiring ischemic surgery such as acute mesenteric ischemia, acute ischemic conditions such as peripheral artery disease,

- Patients diagnosed with pulmonary embolism.

- Patients with a high risk of chest pain and ST-elevation MI,

- Patients with chest pain accompanied by hypotension, new ECG changes, confusion.

- Patients with a history of cardiac catheterization within 1 month.

- Patients diagnosed with myocardial infarction within 1 month.

- Patients whose data cannot access through the hospital information system.

In the study hospital, traditionally, the patients with chest pain suspected AMI are undergone serial troponin test at $0 \mathrm{~h}, 3 \mathrm{~h}$, and $6 \mathrm{~h}$. For low-risk patients or patients who have had chest pain for more than $6 \mathrm{~h}$, the discharge decision was made with $0 \mathrm{~h}$ and $3 \mathrm{~h}$ serial troponin. In this study, we added $1 \mathrm{~h}$ and $2 \mathrm{~h}$ serial troponin testing to this traditional process.

\section{Data collection}

The data were collected via the emergency physicians in the study hospital into the patient record which age, gender, history, characteristics of pain, vital signs in the triage room 
Patients $\geq 18$ years with chest pain and investigated for $\mathrm{AMI}(\mathrm{n}=2100)$

Unstable vital signs

Investigating for any other diagnosis,

trauma, pulmnary embolism, aortic

dissection $(n=700)$

History of cardiac catheterization

/CABG within one month(n=15)

Ongoing chest pain $(\mathrm{n}=135)$

Other chest pain diagnosis* than AMI on

imaging and laboratory tests $(\mathrm{n}=116)$

Patients with STEMI $(\mathrm{n}=45)$,

ECG changes on follow-up( $(n=7)$,

New abnormal vital signs on follow

$\operatorname{up}((\mathrm{n}=5)$

Data not accessed( $n=3)$.

hsTnI Elevation $<99$ percentile $(\mathrm{n}=163)$

Follow up with serial hs $\operatorname{TnI}$ on 0., 1., 2. hour and $\mathrm{ECG}(\mathrm{n}=911)$

Fig. 1 Selection of the patients enrolled in the study. Ischemic/hemorrhagic cerebrovascular event, acute renal failure, requiring ischemic surgery such as acute mesenteric ischemia, acute ischemic conditions such as perpheral artery disease, pulmonary embolism

were first recorded real-time at admission. After the physician decided that the patient has a suspected ACS (Fig. 2) are undergone serial troponin follow-up as $0 \mathrm{~h}, 1 \mathrm{~h}, 2 \mathrm{~h}, 3 \mathrm{~h}$ (and $6 \mathrm{~h}$ if necessary regarding the onset of chest pain). During the follow-up, the other diagnoses are discarded with echocardiography, pulmonary angiography, and other laboratory tests. The patients' hsTnI on admission, in the 1st hour (early time point for COMPASS-MI), 2nd hour (late timepoint for COMPASSMI), 3rd-hour values were measured and recorded. EDACSADP and COMPASS-MI risk scores were calculated according to the relevant studies $[6,10]$. Also, the consultation and outcome of the patient were recorded on the same day. If the troponin levels are higher than the cutoff levels, the cardiologist decides admission to the hospital. Thirty-day after ED visit, MACE (MI, CABG or PCI, death) and mortality were acquired by one of the study researchers via accessing the hospital information system or by patient phone whether any cardiac adverse events or death occurred within 30 days. 
The patient with chest pain

Does the patient has the exclusion criteria?

- Patients with traumatic chest pain

- Patients with suspected or detected COVID-19 in the study period

- Patients with a high risk of chest pain(the same pain in the AMI history) and ST-elevation MI

- Patients with chest pain accompanied by hypotension, confusion.

- Patients with a history of cardiac catheterization within one month

- Patients diagnosed with myocardial infarction within one month

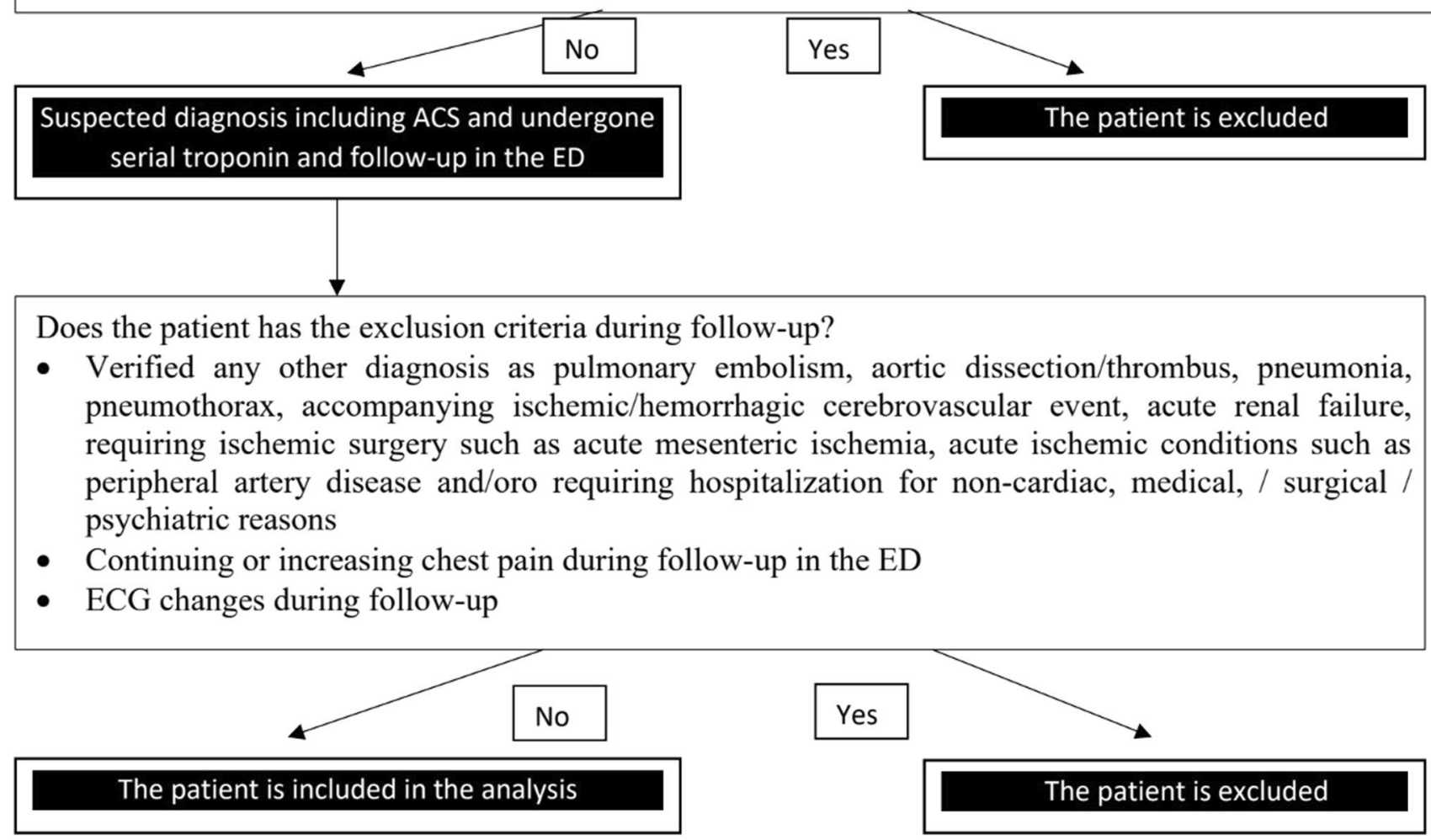

Fig. 2 Algorithm of the study protocol of the patients included in the study regarding the progress in the emergency department

\section{Measures}

\section{hsTroponin I and COMPASS-MI calculator}

Hs-TnI was measured using the Backman Coulter Access Hs-TnI assay, which has an overall 99th percentile of $17 \mathrm{ng} / \mathrm{L}$ with a coefficient of variation $(\mathrm{CV})$ of $<5 \%$ and a limit of detection of $1.9 \mathrm{ng} / \mathrm{L}$.

The Access hsTnI assay cutoff is defined as $17.5 \mathrm{pg} / \mathrm{mL}$ (ng/L) with a 95\% confidence interval (CI) of 12.6-20.7 pg/ $\mathrm{mL}(\mathrm{ng} / \mathrm{L})$ with a level of detection $2.3 \mathrm{pg} / \mathrm{mL}(\mathrm{ng} / \mathrm{L})$.

In the calculation of the COMPASS-MI risk tool, we used the original calculator from the given study [6] and defined the change in the $0 \mathrm{~h}$ and $1 \mathrm{~h}$ hsTnI levels as "change in the early-stage timepoint" and the change in the $0 \mathrm{~h}$ and $2 \mathrm{~h}$ hsTnI levels as "change in the late-stage timepoint." After we used the cutoff level as defined in the assay $17.5 \mathrm{ng} / \mathrm{ml}$ and the change in the hsTnI, the appropriate risk group was chosen for the patient. We used the same hsTnI assay in the EDACS-ADP score.

\section{Data analysis}

All analyses were performed on SPSS v21 (SPSS Inc., Chicago, IL, USA). Q-Q and histogram plots were used to determine whether variables are normally distributed. 
Data are given as mean \pm standard deviation or median (1st quartile-3rd quartile) for continuous variables according to the normality of distribution and frequency (percentage) for categorical variables. Normally distributed variables were analyzed with the independent samples $t$ test. Non-normally distributed variables were analyzed with the Mann-Whitney $U$ test. Categorical variables were analyzed with the chi-squared test or Fisher's exact test. MACE prediction performance of the variables was evaluated using Receiver Operating Characteristic (ROC) curve analysis. Multiple logistic regression analyses (conditional forward method) were performed to determine the best predictive factors of the 30-day MACE. Two-tailed $p$ values of less than 0.05 were considered statistically significant. Sensitivity, specificity, NPV, PPV are defined for the performance of the tools.

\section{Effect of size}

We calculated the effect of size on EDACS-ADP score for both MACE and non-MACE groups. The mean EDACSADP score was 9.43(95\% CI 8.94-9.92) \pm 7.33 for the nonMACE group and 16.24 (95\% CI 13.64-18.83) \pm 7.90 for the MACE group. Cohen's d was -0.893 (r-0.407).

\section{Results}

We included 911 patients (385 females and 526 males) in our study. The mean age was $49.49 \pm 15.91$ (range 18-94). Thirty-eight (14.17\%) patients had significant adverse cardiovascular events (MACE) within 30 days; 2 of them were mortal. Thirty-five patients (3.7\%) were admitted to the cardiology clinic for further investigations, $96.2 \%(n=876)$ of all patients were discharged.

Age was significantly higher in the MACE group than in the non-MACE group $(p<0.001)$. Ca channel blockers use $(p=0.027)$ and ACE inhibitors use $(p=0.001)$ percentages were significantly higher in the MACE group than in the non-MACE group. There were no significant differences between groups concerning gender and smoking percentages (Table 1).

Coronary artery disease $(p<0.001)$, heart failure $(p=0.006)$, hypertension $(p=0.011)$, and diabetes mellitus $(p=0.034)$ percentages were significantly higher in the MACE group than in the non-MACE group. There were $20(52.63 \%)$ patients with known coronary artery disease or $\geq$ three risk factors in the MACE group, while there were $192(21.99 \%)$ patients with known coronary artery disease or $\geq$ three risk factors in the non-MACE group $(p<0.001)$.

Duration of symptoms was significantly higher in the non-MACE group than in the MACE group $(p=0.012)$. The percent of the symptoms less than $4 \mathrm{~h}$ was $55.9 \%$ $(n=589)$. Forty-two patients had chest pain for more than
1 week. Blunt (Burning/Chest heaviness/Squeezing/Pressure-like chest pain) percentage was significantly higher in the MACE group than in the non-MACE group $(p<0.001)$ (Table 2).

Abnormal chronic changes in electrocardiogram ( $p<0.001)$, left bundle branch block $(p=0.011)$, ventricular extrasystole $(p=0.001)$, negative T wave $(p=0.003)$, and ST depression $(p=0.026)$ percentages were significantly higher in the MACE group than in the non-MACE group. Normal echocardiography percentages were significantly higher in the non-MACE group than in the MACE group $(p<0.001)$. All CK-MB and troponin measurements $(0 \mathrm{~h}, 1 \mathrm{~h}, 2 \mathrm{~h})$ were significantly higher in the MACE group than in the non-MACE group. Change in $0-1 \mathrm{~h}(p=0.001)$, change in $0-2 \mathrm{~h}$ troponin $(p<0.001)$, EDACS-ADP score $(p<0.001)$, early-stage risk of COMPASS-MI (changes in $1 \mathrm{~h} \mathrm{hsTnI}$ ) $(p=0.008)$, and late-stage risk of COMPASS-MI (changes in $2 \mathrm{~h}$ hsTnI) were significantly higher in the MACE group than in the non-MACE group.

Twenty-one (55.26\%) patients were not low risk according to EDACS-ADP in the MACE group, and 195 (22.34\%) patients were not-low risk in the non-MACE group $(p<0.001)$. Twenty-one $(55.26 \%)$ patients were high risk according to the early-stage risk of COMPASS-MI (changes in $1 \mathrm{~h} \mathrm{hsTnI)} \mathrm{in} \mathrm{the} \mathrm{MACE} \mathrm{group,} \mathrm{and} \mathrm{355(40.66 \% )} \mathrm{patients}$ were high risk in the non-MACE group $(p=0.105)$. Thirty (78.95\%) patients were high risk according to the latestage risk of COMPASS-MI in the MACE group, and 436 $(49.94 \%)$ patients were high risk in the non-MACE group $(p=0.001)$.

When we evaluate the risk of MACE, patients with notlow-risk EDACS-ADP have a 3.975 (95\% CI 2.136-7.396) fold higher risk of MACE than the patients with low-risk EDACS-ADP, and the absolute risk increase is $7.3 \%$ in our study population. In addition, if we have 13.74 patients with not-low-risk EDACS-ADP, we will have one patient with MACE. Patients with high-risk late-stage risk in COMPASS-MI have a 3.581 (95\% CI 1.660-7.726) fold higher risk of MACE than those with low-risk late-stage risk in COMPASS-MI, and absolute risk increase is $4.6 \%$. If we have 21.55 patients with high-risk late-stage risk in COMPASS-MI, we will have one patient with MACE (Table 3).

When we evaluated the MACE prediction performance of the scoring systems, we found EDACS-ADP has the highest specificity, accuracy, positive predictive value, and area under the ROC curve. In contrast, the late-stage risk of COMPASS-MI has the highest sensitivity and negative predictive value. EDACS-ADP $(p=0.001)$ and late-stage risk of COMPASS-MI $(p=0.002)$ were found statistically significant regarding MACE prediction performances. In addition, all negative predictive values were above 95\% (Table 3).

The patients with low late-stage risk of COMPASS-MI risk and low-risk EDACS-ADP score have statistically lower 
Table 1 Summary of patients and symptom characteristics with regard to 30-day MACE

\begin{tabular}{|c|c|c|c|c|}
\hline & \multicolumn{2}{|l|}{ 30-day MACE } & \multirow[t]{2}{*}{ Total $(n=911)$} & \multirow[t]{2}{*}{$p$} \\
\hline & Absent $(n=873)$ & Present $(n=38)$ & & \\
\hline Age & $48.93 \pm 15.77$ & $62.21 \pm 13.96$ & $49.49 \pm 15.91$ & $<0.001$ \\
\hline \multicolumn{5}{|l|}{ Sex } \\
\hline Female & $370(42.38 \%)$ & $15(39.47 \%)$ & $385(42.26 \%)$ & \multirow[t]{2}{*}{0.851} \\
\hline Male & $503(57.62 \%)$ & $23(60.53 \%)$ & $526(57.74 \%)$ & \\
\hline Smoking & $204(23.37 \%)$ & $4(10.53 \%)$ & $208(22.83 \%)$ & 0.099 \\
\hline Beta blockers & $139(15.92 \%)$ & $11(28.95 \%)$ & $150(16.47 \%)$ & 0.058 \\
\hline Ca channel blockers & $66(7.56 \%)$ & $7(18.42 \%)$ & $73(8.01 \%)$ & 0.027 \\
\hline ACE inhibitors & $118(13.52 \%)$ & $13(34.21 \%)$ & $131(14.38 \%)$ & 0.001 \\
\hline Antihyperlipidemic & $77(8.82 \%)$ & $5(13.16 \%)$ & $82(9.00 \%)$ & 0.378 \\
\hline Acetylsalicylic acid & $142(16.27 \%)$ & $11(28.95 \%)$ & $153(16.79 \%)$ & 0.068 \\
\hline Clopidogrel & $51(5.84 \%)$ & $4(10.53 \%)$ & $55(6.04 \%)$ & 0.281 \\
\hline Oral anticoagulants & $50(5.73 \%)$ & $3(7.89 \%)$ & $53(5.82 \%)$ & 0.480 \\
\hline Antidiabetics & $82(9.39 \%)$ & $3(7.89 \%)$ & $85(9.33 \%)$ & 1.000 \\
\hline Family history & $42(4.81 \%)$ & $3(7.89 \%)$ & $45(4.94 \%)$ & 0.428 \\
\hline Obesity & $5(0.57 \%)$ & $0(0.00 \%)$ & $5(0.55 \%)$ & 1.000 \\
\hline Coronary artery disease & $183(20.96 \%)$ & $20(52.63 \%)$ & $203(22.28 \%)$ & $<0.001$ \\
\hline CABG & $42(4.81 \%)$ & $4(10.53 \%)$ & $46(5.05 \%)$ & 0.119 \\
\hline Heart failure & $15(1.72 \%)$ & $4(10.53 \%)$ & $19(2.09 \%)$ & 0.006 \\
\hline Valvular heart disease & $13(1.49 \%)$ & $0(0.00 \%)$ & $13(1.43 \%)$ & 1.000 \\
\hline Hypertension & $200(22.91 \%)$ & $16(42.11 \%)$ & $216(23.71 \%)$ & 0.011 \\
\hline Diabetes mellitus & $113(12.94 \%)$ & $10(26.32 \%)$ & $123(13.50 \%)$ & 0.034 \\
\hline Chronic renal disease & $17(1.95 \%)$ & $2(5.26 \%)$ & $19(2.09 \%)$ & 0.186 \\
\hline Cerebrovascular disease & $10(1.15 \%)$ & $1(2.63 \%)$ & $11(1.21 \%)$ & 0.376 \\
\hline Hyperlipidemia & $76(8.71 \%)$ & $3(7.89 \%)$ & $79(8.67 \%)$ & 1.000 \\
\hline Malignancy & $10(1.15 \%)$ & $0(0.00 \%)$ & $10(1.10 \%)$ & 1.000 \\
\hline Known $\mathrm{CAD}$ or $\geq 3$ risk factors & $192(21.99 \%)$ & $20(52.63 \%)$ & $212(23.27 \%)$ & $<0.001$ \\
\hline \multicolumn{5}{|l|}{ Symptoms } \\
\hline N/A & $533(61.1 \%)$ & $27(71.1 \%)$ & $560(61.5 \%)$ & \\
\hline Diaphoresis & $19(2.2 \%)$ & $3(7.9 \%)$ & $22(2.4 \%)$ & 0.107 \\
\hline Pain occurred or worsened with inspiration & $203(23.3 \%)$ & $7(18.4 \%)$ & $210(23.1 \%)$ & 0.296 \\
\hline Pain is reproduced by palpation & $78(8.9 \%)$ & $1(2.6 \%)$ & $79(8.7 \%)$ & \multirow[t]{2}{*}{0.077} \\
\hline At least 2 of the above & $40(4.6 \%)$ & $0(0.0 \%)$ & $40(4.4 \%)$ & \\
\hline Pain radiation & $166(19.01 \%)$ & $10(26.32 \%)$ & $176(19.32 \%)$ & \multirow[t]{6}{*}{0.365} \\
\hline Arm & $87(9.97 \%)$ & $3(7.89 \%)$ & $90(9.88 \%)$ & \\
\hline Chin & $16(1.83 \%)$ & $0(0.00 \%)$ & $16(1.76 \%)$ & \\
\hline Shoulder & $23(2.63 \%)$ & $2(5.26 \%)$ & $25(2.74 \%)$ & \\
\hline Neck & $13(1.49 \%)$ & $0(0.00 \%)$ & $13(1.43 \%)$ & \\
\hline Back-Scapular & $46(5.27 \%)$ & $5(13.16 \%)$ & $51(5.60 \%)$ & \\
\hline Duration of symptom (h) & $2(1-4)$ & $1(1-2)$ & $2(1-4)$ & 0.012 \\
\hline \multicolumn{5}{|l|}{ Type of symptom } \\
\hline Other & $41(4.70 \%)$ & $2(5.26 \%)$ & $43(4.72 \%)$ & \multirow[t]{3}{*}{$<0.001$} \\
\hline Burning/Chest heaviness/Squeezing/Pressure & $145(16.61 \%)$ & $16(42.11 \%)$ & $161(17.67 \%)$ & \\
\hline Sharp, Stabbing, Pricking & $687(78.69 \%)$ & $20(52.63 \%)$ & $707(77.61 \%)$ & \\
\hline
\end{tabular}

Data are given as mean \pm standard deviation or median (1st quartile-3rd quartile) for continuous variables according to the normality of distribution and as frequency (percentage) for categorical variables 
Table 2 Summary of physical examination findings, laboratory measurements, and outcome with regard to 30-day MACE

\begin{tabular}{|c|c|c|c|c|}
\hline & \multicolumn{2}{|l|}{ 30-day MACE } & \multirow[t]{2}{*}{ Total $(n=911)$} & \multirow[t]{2}{*}{$p$} \\
\hline & Absent $(n=873)$ & Present $(n=38)$ & & \\
\hline Systolic blood pressure & $130(121-136)$ & $132(121-143)$ & $130(121-136)$ & 0.304 \\
\hline Diastolic blood pressure & $79(74-87)$ & $78.5(76-88)$ & $79(74-87)$ & 0.909 \\
\hline Pulse & $81.63 \pm 14.01$ & $82.26 \pm 16.15$ & $81.66 \pm 14.09$ & 0.787 \\
\hline Oxygen saturation & 97 (96-98) & $96.5(96-98)$ & 97 (96-98) & 0.364 \\
\hline Abnormal ECG & $108(12.37 \%)$ & $19(50.00 \%)$ & $127(13.94 \%)$ & $<0.001$ \\
\hline LBBB & $18(2.06 \%)$ & $4(10.53 \%)$ & $22(2.41 \%)$ & 0.011 \\
\hline VES & $9(1.03 \%)$ & $4(10.53 \%)$ & $13(1.43 \%)$ & 0.001 \\
\hline Negative $T$ wave & $31(3.55 \%)$ & $6(15.79 \%)$ & $37(4.06 \%)$ & 0.003 \\
\hline RBBB & $22(2.52 \%)$ & $2(5.26 \%)$ & $24(2.63 \%)$ & 0.264 \\
\hline ST depression & $13(1.49 \%)$ & $3(7.89 \%)$ & $16(1.76 \%)$ & 0.026 \\
\hline Atrial fibrillation & $21(2.41 \%)$ & $2(5.26 \%)$ & $23(2.52 \%)$ & 0.249 \\
\hline \multicolumn{5}{|l|}{ Echocardiography } \\
\hline Not applied & $769(88.09 \%)$ & $24(63.16 \%)$ & $793(87.05 \%)$ & $<0.001$ \\
\hline Normal (Normal or not new abnormal ecocardiographic findings) & $90(10.31 \%)$ & $9(23.68 \%)$ & $99(10.87 \%)$ & \\
\hline $\begin{array}{l}\text { Abnormal findings (newly occurred abnormal findings, low EF, } \\
\text { hypokinetic/akinetic, aneurysm) }\end{array}$ & $14(1.60 \%)$ & $5(13.16 \%)$ & $19(2.09 \%)$ & \\
\hline \multicolumn{5}{|l|}{ CK-MB } \\
\hline 0 hour & $1.6(1.1-2.5)$ & $1.95(1.5-3.1)$ & $1.6(1.1-2.5)$ & 0.004 \\
\hline 1 hour & $1.6(1.1-2.5)$ & $2.15(1.4-3.8)$ & $1.6(1.1-2.5)$ & 0.002 \\
\hline 2 hour & $1.5(1.1-2.5)$ & $2.35(1.4-4.1)$ & $1.6(1.1-2.5)$ & $<0.001$ \\
\hline \multicolumn{5}{|l|}{ Troponin } \\
\hline 0 hour & $3(2-5)$ & $9.5(3-33)$ & $3(2-5)$ & $<0.001$ \\
\hline 1 hour & $3(2-5)$ & $12.5(3-40)$ & $3(2-5)$ & $<0.001$ \\
\hline 2 hour & $3(2-5)$ & $14(3-95)$ & $3(2-5)$ & $<0.001$ \\
\hline Change in early-stage troponin & $0(0-0)$ & $0(0-3)$ & $0(0-0)$ & 0.001 \\
\hline Change in late-stage troponin & $0(0-1)$ & $1(0-20)$ & $0(0-1)$ & $<0.001$ \\
\hline EDACS & $9.43 \pm 7.34$ & $16.24 \pm 7.90$ & $9.72 \pm 7.48$ & $<0.001$ \\
\hline Low & $678(77.66 \%)$ & $17(44.74 \%)$ & $695(76.29 \%)$ & $<0.001$ \\
\hline Not-low & $195(22.34 \%)$ & $21(55.26 \%)$ & $216(23.71 \%)$ & \\
\hline Early stage COMPASS-MI & $0.3(0.3-2.4)$ & $2.4(0.3-3)$ & $0.3(0.3-2.4)$ & 0.008 \\
\hline Low & $518(59.34 \%)$ & $17(44.74 \%)$ & $535(58.73 \%)$ & 0.105 \\
\hline High & $355(40.66 \%)$ & $21(55.26 \%)$ & $376(41.27 \%)$ & \\
\hline Late stage COMPASS-MI & $1.7(0.2-1.7)$ & $1.7(1.7-3.1)$ & $1.7(0.2-1.7)$ & $<0.001$ \\
\hline Low & $437(50.06 \%)$ & $8(21.05 \%)$ & $445(48.85 \%)$ & 0.001 \\
\hline High & $436(49.94 \%)$ & $30(78.95 \%)$ & $466(51.15 \%)$ & \\
\hline Consultation & $256(29.32 \%)$ & $30(78.95 \%)$ & $286(31.39 \%)$ & $<0.001$ \\
\hline Discharge & $239(27.38 \%)$ & $13(34.21 \%)$ & $252(27.66 \%)$ & $<0.001$ \\
\hline Hospitalization & $17(1.95 \%)$ & $17(44.74 \%)$ & $34(3.73 \%)$ & \\
\hline \multicolumn{5}{|l|}{ Final status } \\
\hline Discharge & $856(98.05 \%)$ & $20(52.63 \%)$ & $876(96.16 \%)$ & \\
\hline Admitted to Cardiology in-patient clinic & $17(1.94 \%)$ & $18(47.37 \%)$ & $35(3.84 \%)$ & \\
\hline \multicolumn{5}{|l|}{ 30-day MACE } \\
\hline 30-day Mortality (All discharged from ED) & $0(0.00 \%)$ & $2(5.26 \%)$ & $2(0.22 \%)$ & 0.002 \\
\hline
\end{tabular}

Data are given as mean \pm standard deviation or median (1st quartile-3rd quartile) for continuous variables according to the normality of distribution and as frequency (percentage) for categorical variables 
Table 3 Risk of 30-day MACE prediction performance of the scoring systems

\begin{tabular}{llll}
\hline & EDACS & \multicolumn{2}{l}{ COMPASS-MI } \\
\cline { 3 - 4 } \cline { 3 - 4 } & & Early stage & Late stage \\
\hline Relative risk (95\% CI) & $3.975(2.136-7.396)$ & $1.758(0.940-3.286)$ & $3.581(1.660-7.726)$ \\
Absolute risk increase & 0.073 & 0.024 & 0.046 \\
The number needed to harm & 13.74 & 41.54 & 21.55 \\
Sensitivity & $55.26 \%$ & $55.26 \%$ & $78.95 \%$ \\
Specificity & $77.66 \%$ & $59.34 \%$ & $50.06 \%$ \\
Accuracy & $76.73 \%$ & $59.17 \%$ & $51.26 \%$ \\
Positive predictive value & $9.72 \%$ & $5.59 \%$ & $6.44 \%$ \\
Negative predictive value & $\mathbf{9 7 . 5 5 \%}$ & $96.82 \%$ & $\mathbf{9 8 . 2 0} \%$ \\
AUC (95\% CI) & $0.665(0.570-0.760)$ & $0.573(0.480-0.666)$ & $0.645(0.563-0.727)$ \\
$p$ & 0.001 & 0.127 & 0.002 \\
\hline
\end{tabular}

$C I$ confidence interval, $A U C$ area under ROC curve, $C I$ confidence intervals
30-day-MACE in all age groups $(p=0.04)$ in our study population.

When we evaluated the best predictive factors, we performed multiple logistic regression analyses to determine the best predictive factors of the 30-day MACE. We found that high age $(p<0.001)$ and high change in late-stage Troponin $(p<0.001)$ are the best predictive factors of the MACE (Table 4). Other variables included in the model, gender $(p=0.444)$, drug use $(p=0.250)$, presence of risk factor $(p=0.269)$, baseline troponin level $(p=0.910)$, earlystage troponin level $(p=0.952)$, late-stage troponin level $(p=0.910)$, change in early-stage troponin $(p=0.434)$, EDACS-ADP ( $p=0.508)$ were found to be non-significant. In subgroup analysis, when we measure the specificity, sensitivity, NPV, PPV of the patients according to their EDACSADP groups, we found the best NPV as $99 \%$ in low-risk EDACS-ADP patients under the LOD after $2 \mathrm{~h}$ hsTnI but low sensitivity and specificity as $76 \%, 46 \%$, respectively. It also has a high NPV of $96 \%$ in high-risk EDACS-ADP patients with a sensitivity of $95 \%$. Among the subgroups under the cutoff $(17.5 \mathrm{ngn} / \mathrm{ml})$, the NPV of $2 \mathrm{~h}$ hsTnI was showed $92 \%$ and $96 \%$ of sensitivity and NPV in high-risk EDACS-ADP patients.

When we tested the discharge decision using $0 \mathrm{~h}$ hsTnI, the best performance was $98 \%$ PPV and $96 \%$ sensitivity using cutoff levels $(17.5 \mathrm{pg} / \mathrm{ml})$ of hsTnI (Supp1).

\section{Discussion}

Discharging a patient safely from the emergency department is a critical decision for an emergency physician. To avoid misdiagnosing AMI, which is one of the most common causes of death, timing and retesting are the significant complexities for decision-making to safely and easily discharge. This study aimed to investigate the performances of EDACS-ADP with change in the hsTnI baseline $1 \mathrm{~h}$ and $2 \mathrm{~h}$ using COMPASS-MI risk calculator to predict 30-days mortality. In the low-risk patients classified with EDACS-ADP, the risk classification with COMPASS-MI calculator using hsTnI, the negative predictive values are found $98 \%$. We aimed to compare these two tools, clinical and biochemical, and the other is only biochemical in our population.

In a meta-analysis of evaluating the accuracy of EDACS-ADP score by Boyle et al. [11], the overall sensitivity and specificity were $96.1 \%$ and $61.1 \%$, respectively. On the other hand, the original EDACS-ADP study by Than et al. showed that EDACS-ADP has a sensitivity of $99 \%$ and specificity of $49.9 \%$. We found EDACS-ADP score has a sensitivity and specificity as $55.26 \%$ and $77.66 \%$, respectively. This difference in sensitivity and specificity may be due to low prevalence of MACE and
Table 4 Significant predictive factors of the 30-day MACE, multiple logistic regression analysis

\begin{tabular}{|c|c|c|c|c|c|c|}
\hline \multirow[b]{2}{*}{ Age } & \multirow{2}{*}{$\frac{\beta \text { coefficient }}{0.048}$} & \multirow{2}{*}{$\begin{array}{l}\text { Standard error } \\
0.012\end{array}$} & \multirow{2}{*}{$\begin{array}{l}p \\
<0.001\end{array}$} & \multirow{2}{*}{$\begin{array}{l}\operatorname{Exp}(\beta) \\
1.049\end{array}$} & \multicolumn{2}{|c|}{$\begin{array}{l}95 \% \text { CI for } \operatorname{Exp} \\
(\beta)\end{array}$} \\
\hline & & & & & 1.024 & 1.074 \\
\hline $\begin{array}{l}\text { Change in late- } \\
\text { stage troponin }\end{array}$ & 0.011 & 0.003 & $<0.001$ & 1.011 & 1.006 & 1.017 \\
\hline (Constant) & -5.980 & 0.748 & $<0.001$ & 0.003 & & \\
\hline
\end{tabular}

Cox \& Snell $R^{2}=0.061 ;$ Nagelkerke $R^{2}=0.208$; Overall percentage $=96.16 \%$

CI Confidence Interval 
low prevalence of not-low-risk patients in our population. Mariska et al. also interpreted this variation with 23 metaanalyses and stated that the changes in the prevalence from 1 to $77 \%$ may cause a difference up to $40 \%$ in sensitivity [12]. Higher disease prevalences make lower specificity [12]. The patients with low risk for EDACS-ADP showed a rate of $1.9 \%$ at MACE. This rate was found $0.54 \%$ in the meta-analysis. This also explains why our specificity of MACE prediction is lower in our low-risk patients.

The original study of the COMPASS-MI tool [6] has reported the risk estimation of MI via hsTnI concentrations, serial sampling, and cutoff levels of the assay regarding MACE and death at 30 days. The probabilities help decide discharge or follow-up in ED. The patients with chest pain are often scored and triaged regarding their risk of MACE to whether follow-up in the ED or safely discharge. The EDACS-ADPEDACS-ADP risk score is used for assessing the chest pain features and the patient risk factors in addition to 2-h troponin levels [10]. Besides, the COMPASS-MI score is also a tool for estimating the risk for MI and death only via cutoff levels of high-sensitivity Troponin [6]. Thus occurred whether to use absolute change from $0 \mathrm{~h}$ to $2 \mathrm{~h}$ or elevation at the $2 \mathrm{~h}$ of hsTnI (equivalent to late-stage timepoint for COMPASS-MI) to improve the performance of the tests. Our study evaluated and used these two calculations and found that patients can be discharged with low risk at a $97.55 \%$ NPV with EDACS-ADP score and $98.20 \%$ with COMPASS-MI risk estimation with a late time point ( $2 \mathrm{~h}-120 \mathrm{~min}$ hsTn).

Another rule-out strategy was $0 \mathrm{~h}$ and $1 \mathrm{~h} \mathrm{hsThI}$ as defined in the European Society of Cardiology [13, 14]. Since the guideline recommends the hsTnI and hsTnT levels for defined assays, the patient with a hsTnI $<2$ at admission or $<5$ at admission with a change $<2$ at $1 \mathrm{~h}$ could be discharged safely. Mokhtari et al. adapted this strategy to TIMI and ECG changes and found an NPV of 99.5\% and an LR of 0.04 for 30-day MACE [15]. We also evaluated the $0 \mathrm{~h}$ and $1 \mathrm{~h}$ for early discharge of COMPASS-MI and rule-out strategy; the MACE did not statistically change in our population.

Besides the difference of assays, Carlton et al. have studied the detection level for ruling out ACS [9]. In parallel to the study, in the low-risk EDACS-ADP group, the LOD (level of detection) cutoff showed the best negative predictive values at $99 \%$.

EDACS-ADP-ADP is a more than $98.7 \%$ sensitive tool for discharging patients with low risk and negative troponin levels [10]. Our study is still sensitive and parallel to the original study, which had not achieved the target. EDACS-ADP.

Most of the elevated troponin etiologies are renal failure, sepsis, other thromboembolic events. Further studies should also be initiated and create cutoff levels used for the discharge of these patients.

\section{Limitations}

Our first limitation is one center cohort with a sample size of nine hundred eleven patients. Since the Caucasian EDACSADP score cohort was a study in which elderly patients were predominant, $17 \%$ of the patients in our study were 65 years of age or older. Nearly $25 \%$ of the cohort had known coronary artery disease or $\geqq 3$ risk factors. In EDACS-ADP, this criteria got one of the highest points when calculating the risk score. Regarding the external validity of this study to other populations, $96 \%$ of patients were discharged from the ED, which may suggest the population of the study as low risk. This result is due to the triage of the patients for exclusion from the beginning. We excluded all the STEMI, continuing chest pain, accompanying other diagnoses, and unstable vital signs in the triage. Although the EDACS-ADP score was made in patients with normal vital signs, there are also patients with abnormal vital signs, hypertension as a chronic risk factor; the patients with HT have high blood pressure at presentation but are not unstable or not causing any end-organ injury. We believe the patients with risk factors also visit emergency departments with chest pain other than acute coronary syndrome. Patients with a history of CAD and comorbid diseases are the most significant independent risk factors. Thus, there is a need for further clear studies for patients between gray zone for comorbid diseases.

Further studies may include recurrent visits for chest pain. The troponin assays are different from original studies, which debates the reliability of the scores. The assay differences between our study and the original COMPASSMI study are also debated, especially at their different cutoff levels.

\section{Conclusions}

Our study evaluated and used these two calculations and found that patients can be categorized as low risk at a 97.55\% NPV with EDACS-ADP score and $98.20 \%$ with COMPASS-MI risk estimation at late timepoint ( $2 \mathrm{~h}-120$ min hsTn) in our population.

\section{Declarations}

Conflict of interest The authors declare that they have no conflict of interest. 


\section{References}

1. Smith LM, Mahler SA (2020) Chest pain. Tintinalli's emergency medicine. McGraw Hill, New York, pp 329-333

2. Gibbs J, deFilippi C, Peacock F, Mahler S, Nowak R, Christenson R, Apple F, Jacobsen G, McCord J (2020) The utility of risk scores when evaluating for acute myocardial infarction using highsensitivity cardiac troponin I. Am Heart J 227:1-8

3. Miller C, Granger CB, Hoekstra J, Jaffe AS (2021) Evaluation of emergency department patients with chest pain at low or intermediate risk for acute coronary syndrome. In: UpToDate, Dardas TF (Ed), UpToDate, Waltham, MA. (Accessed on December 29, 2021.)

4. Antman EM, Cohen M, Bernink PJLM, McCabe CH, Horacek T, Papuchis G, Mautner B, Corbalan R, Radley D, Braunwald E (2000) The TIMI risk score for unstable angina/non-ST elevation MI: a method for prognostication and therapeutic decision making. JAMA 284:835

5. Six AJ, Backus BE, Kelder JC (2008) Chest pain in the emergency room: value of the HEART score. Neth Heart J 16:191-196

6. Neumann JT, Twerenbold R, Ojeda F, Sörensen NA, Chapman AR, Shah ASV, Anand A, Boeddinghaus J, Nestelberger T, Badertscher P, Makhtari A, Pickering JW, Troughton RW, Greenslade J, Parsonage W, Mueller-Hennessen M, Gori T, Jernberg T, Morris N, Liebetrau C, Hamm C, Katus HA, Münzel T, Landmesser U, Salomaa V, Iacoviello L, Ferrario MM, Giampaoli S, Kee F, Thorand B, Peters A, Borchini R, Jorgensen T, Söderberg S, Sans S, Tunstall-Pedoe H, Kuulasmaa K, Renne T, Lackner KJ, Worster A, Body R, Ekelund U, Kavsak PA, Keller T, Lindahl B, Wild P, Giannitsis E, Than M, Cullen LA, Mills NL, Mueller C, Zeller T, Westermann D, Blankenberg S (2019) Application of highsensitivity troponin in suspected myocardial infarction. N Engl J Med 380:2529-2540

7. Barnes C, Fatovich DM, Macdonald SPJ, Alcock RF, Spiro JR, Briffa TG, Schultz CJ, Hillis GS (2021) Single high-sensitivity troponin levels to assess patients with potential acute coronary syndromes. Heart 107:721-727

8. Shah ASV, Anand A, Sandoval Y, Lee KK, Smith SW, Adamson PD, Chapman AR, Langdon T, Sandeman D, Vaswani A, Strachan FE, Ferry A, Strizaker AG, Reid A, Gray AJ, Collinson PO, McAllister DA, Apple FS, Newby DE, Mills NL (2015) High-sensitivity cardiac troponin I at presentation in patients with suspected acute coronary syndrome: a cohort study. Lancet 386:2481-2488
9. Carlton EW, Ingram J, Taylor H, Glynn J, Kandiyali R, Campbell S, Beasant L, Aziz S, Beresford P, Kendall J, Reuben A, Smith JE, Chapman R, Creanor S, Benger JR (2020) Limit of detection of troponin discharge strategy versus usual care: randomised controlled trial. Heart 106:1586-1594

10. Than M, Flaws D, Sanders S, Dooust J, Glasziou P, Kline J, Aldous S, Troughton R, Reid C, Parsonage WA, Frampton C, Greenslade JH, Deely J, Hess E, Sadiq AB, Singleton R, Shopland R, Vercoe L, Woolhouse-Williams M, Ardagh M, Bossuyt P, Bannister L, Culllen L (2014) Development and validation of the emergency department assessment of chest pain score and 2 $\mathrm{h}$ accelerated diagnostic protocol: emergency department assessment of chest pain score. Emerg Med Australas 26:34-44

11. Boyle RSJ, Body R (2021) The diagnostic accuracy of the emergency department assessment of chest pain (EDACS) score: a systematic review and meta-analysis. Ann Emerg Med 77:433-441

12. Leeflang MMG, Rutjes AWS, Reitsma JB, Hooft L, Bossuyt PMM (2013) Variation of a ' 'test's sensitivity and specificity with disease prevalence. CMAJ 185:E537-E544

13. Roffi M, Patrono C, Collet J-P, Mueller C, Valgimigli M, Andreotti F, Bax JJ, Borger MA, Brotons C, Chew DP, Gencer B, Hasenfuss G, Kjeldsen K, Lancelotti P, Landmesser U, Mehilli J, Mukherjee D, Storey RF, Windecker S (2016) 2015 ESC Guidelines for the management of acute coronary syndromes in patients presenting without persistent ST-segment elevation: task force for the management of acute coronary syndromes in patients presenting without persistent ST-segment elevation of the European society of cardiology (ESC). Eur Heart J 37:267-315

14. Stoyanov KM, Hund H, Biener M, Gandowitz J, Riedle C, Löhr J, Mueller-Hennessen M, Vafaie M, Katus HA, Giannitsis E (2020) RAPID-CPU: a prospective study on implementation of the ESC 0/1-hour algorithm and safety of discharge after rule-out of myocardial infarction. Eur Heart J Acute Cardiovasc Care 9:39-51

15. Mokhtari A, Lindahl B, Schiopu A, Yndigegn T, Khoshnood A, Gilje P, Ekelund U, Diercks DB (2017) A 0-hour/1-hour protocol for safe, early discharge of chest pain patients. Acad Emerg Med 24:983-992

Publisher's Note Springer Nature remains neutral with regard to jurisdictional claims in published maps and institutional affiliations. 